\title{
Serious Adverse Event Reason Type Terminology
}

National Cancer Institute

\section{Source}

National Cancer Institute. Serious Adverse Event Reason Type Terminology. NCI

Thesaurus. Code C105757.

Terminology codelist containing values associated with the rationale for why the adverse event was reported as being serious. 